\title{
Long-Term Results of Bariatric Restrictive Procedures: A Prospective Study
}

\author{
Ruben Schouten • Dorothee C. Wiryasaputra • \\ Francois M. H. van Dielen • Wim G. van Gemert • \\ Jan Willem M. Greve
}

Published online: 19 June 2010

(C) The Author(s) 2010. This article is published with open access at Springerlink.com

\begin{abstract}
Laparoscopic adjustable gastric banding (LAGB) and vertical-banded gastroplasty (VBG) are surgical treatment modalities for morbid obesity. This prospective study describes the long-term results of LAGB and VBG. One hundred patients were included in the study. Fifty patients underwent LAGB and 50 patients, open VBG. Study parameters were weight loss, changes in obesity-related comorbidities, long-term complications, re-operations including conversions to other bariatric procedures and laboratory parameters including vitamin status. From 91 patients (91\%), data were obtained with a mean follow-up duration of 84 months (7 years). Weight loss [percent excess weight loss (EWL)] was significantly more after VBG compared with LAGB, $66 \%$ versus 54\%, respectively. All comorbidities significantly decreased in both groups. Long-term complications after VBG were mainly staple line disruption (54\%) and incisional hernia (27\%). After LAGB, the most frequent complications were pouch dilatation (21\%) and anterior
\end{abstract}

This study was presented in part to the Third Congress of the International Federation for the Surgery of Obesity and Metabolic Disorders-European Chapter, Capri, Italy, April 2008.

This study was presented to the 13th World Congress of the International Federation for the Surgery of Obesity and Metabolic Disorders, Buenos Aires, Argentina, September 2008.

R. Schouten $(\bowtie) \cdot$ W. G. van Gemert $\cdot$ J. W. M. Greve

Department of Surgery, Atrium Medical Centre,

P.O. box 4446, 6401 CX Heerlen, The Netherlands

e-mail: schoutenruben@hotmail.com

D. C. Wiryasaputra

Department of Surgery, Maastricht University Medical Centre,

Maastricht, The Netherlands

F. M. H. van Dielen

Department of Surgery, Maxima Medical Centre,

Veldhoven, The Netherlands slippage (17\%). Major re-operations after VBG were performed in $60 \%$ of patients. All re-operations following were conversions to Roux-en-Y gastric bypass (RYGB). In the LAGB group, $33 \%$ of patients had a refixation or replacement of the band, and $11 \%$ underwent conversion to another bariatric procedure. There were no significant differences in weight loss between patients with or without re-interventions. No vitamin deficiencies were present after 7 years, although supplement usage was inconsistent. This long-term follow-up study confirms the high occurrence of late complications after restrictive bariatric surgery. The failure rate of $65 \%$ after VBG is too high, and this procedure is not performed anymore in our institution. The re-operation rate after LAGB is decreasing as a result of new techniques and materials. Results of the reoperations are good with sustained weight loss and reduction in comorbidities. However, in order to achieve these results, a durable and complete follow-up after restrictive procedures is imperative.

Keywords Restrictive procedures - VBG · LAGB .

Long-term results $\cdot$ Re-operations

\section{Introduction}

Morbid obesity, defined as a body mass index (BMI) of $>40 \mathrm{~kg} / \mathrm{m}^{2}$ or $>35 \mathrm{~kg} / \mathrm{m}^{2}$ with obesity-related comorbidities, is a rapidly growing problem in the western society. In the USA, about 6 million people currently have a BMI of $>40 \mathrm{~kg} / \mathrm{m}^{2}$, and another 10 million people have a BMI of $>35 \mathrm{~kg} / \mathrm{m}^{2}$ with comorbidities. Worldwide, the incidence of morbid obesity has doubled, and about 300 million people are now obese [1, 2]. In The Netherlands, the prevalence is less disturbing, but the incidence of morbid obesity is rising progressively to $1.5 \%$ of the total population [3]. At this moment, surgical 
therapy is the only treatment option for these patients that results in sufficient long-term weight loss. Non-surgical approaches, like low-energy diets and behavior modification, are not successful in the long-term with respect to maintaining weight loss and decreasing obesity-related comorbidities $[4,5]$.

Gastric restrictive surgery was introduced in the mid1970s with transverse gastroplasty and has been developed and improved since. This procedure evolved to verticalbanded gastroplasty (VBG) as described by Mason and others and has been performed on many patients worldwide $[6,7]$. In 1993, a new type of gastric restrictive surgery was introduced in the form of laparoscopic adjustable gastric banding (LAGB) with the advantages of a minimal invasive technique and adjustability and as a result more gradual weight loss [8]. In 1998, no randomized trials were published that compared these two operative techniques. A randomized controlled clinical trial was conducted in our institution by van Dielen et al. The 2-year results of this study demonstrated a significantly shorter hospital stay after LAGB (3.5 versus 6.8 days), a significantly greater weight loss after VBG (\% excess weight loss (EWL) of $72.7 \%$ versus $53.3 \%$ ), significant decrease of all comorbidities in both groups and a higher percentage of major reoperations after VBG (36\% versus 28\%) and incisional hernias (16\% versus $0 \%$ ) [9]. Prospective trials comparing operative techniques are few in bariatric surgery, and only short- or mid-term results are reported [10-12]. Few studies have published long-term results [13, 14]. All patients participating in the aforementioned study have had initial surgery more than 5 years ago. The present study describes the long-term results of this prospectively followed group of patients after restrictive bariatric procedures.

\section{Patients and Methods}

\section{Patients}

Patients were considered eligible for the study if their BMI was $>40$ or $>35 \mathrm{~kg} / \mathrm{m}^{2}$ with obesity-related comorbidities. One hundred patients were included. In the initial study, patients were randomly assigned to LAGB (50) or open VBG (50) using a computer-generated randomization list. The initial study and long-term follow-up study were approved by the medical ethical committee of the Maastricht University Medical Centre, The Netherlands.

\section{Methods}

During the initial study, demographic data, BMI, medication, medical history, and comorbidities were recorded as well as intra- and postoperative complications and length of hospital stay. After discharge, all patients were evaluated in the outpatient clinic after 1 and 6 weeks and after 3,6 , and 12 months. After this period, patients were evaluated once a year. The following data was obtained: body weight, blood pressure, pulse, comorbidities, medication, symptoms and complaints, present dietary habits, complications, reoperations, conversions, and laboratory measurements (hemoglobin, urea, creatinin, aspartate aminotransferase (ASAT), alanine aminotransferase (ALAT), albumin, total protein, cholesterol, triglycerides, high density lipoprotein (HDL) and low density lipoprotein (LDL)). Furthermore, in order to investigate vitamin status and possible deficiencies after restrictive surgery, patients were asked about the use of vitamin supplements and a set of parameters (iron, zinc, vitamin B1, B6, B12, and folic acid) was measured during the last follow-up visit.

\section{Surgical Technique}

All patients received general anesthesia, a single dose of preoperative antibiotics, and a double daily dose of lowmolecular-weight heparin. The surgical techniques of LAGB and open VBG are described in detail elsewhere [9] as well as the technique of converting VBG to RYGB [15]. In summary, in case of VBG, a small pouch of the stomach was created with a 4-row linear stapler (TA-90B, United States Surgical Corp., Norwalk, CT), and a Dacron band of $5.0 \mathrm{~cm}$ in circumference was placed through the window formed by a circular stapler (Premium Plus CEEA $31 \mathrm{~mm}$, United States Surgical Corp., Norwalk, CT). Laparoscopic gastric banding was, in all cases, performed by placing a $9.75-\mathrm{cm}$ lap-band (Allergan, Santa Barbara, CA). During the study period, the LAGB was insufflated when weight loss was insufficient (less than approximately $1 \mathrm{~kg}$ per week) with the first insufflation at least 6 weeks postoperatively. After the first 32 patients, the surgical technique of placing the laparoscopic band was changed from "perigastric" to "pars flaccida" because of several international reports suggesting the latter to be a superior technique with fewer complications [16-18].

\section{Statistical Analysis}

Statistical analyses were performed using the SPSS 15.0.0 statistical package (SPSS Inc., Chicago, IL, USA). The Student's $t$ test was used to calculate differences between the groups. In case of a skewed distribution, the MannWhitney $U$ test was used for comparison between the groups and the Wilcoxon signed-rank test for comparison in one group throughout time. Analysis of the differences in postoperative complications and re-operations between the perigastric and pars flaccida technique in the LAGB group was performed with the Chi-square test. Data are given as mean and standard deviation. A $p$ value of $p<0.05$ was denoted as statistically significant. 


\section{Results}

Preoperative Data

Preoperative patient characteristics are summarized in Table 1. Both groups consisted of 50 patients with a male to female ratio of 1 to 4 . Mean age, weight, BMI, and total number of obesity-related comorbidities did not differ significantly between the groups.

\section{Patients}

Follow-up for the total group was $91 \%$ with a mean period in both groups of 84 months (7 years), ranging from a minimum of 67 months (5.6 years) to a maximum of 101 months ( 8.4 years). In the direct postoperative period, two patients died in the VBG group, one patient due to sepsis as a result of leakage, and one patient due to respiratory complications of a pre-existing pneumonia. Five patients were lost to follow-up. As a result, 43 patients were included in the final analysis in the VBG group. In the LAGB group, two patients died during follow-up as a result of gynecological malignancies. Data from these two deceased patients are included in the analysis until the moment of death. Two patients were lost to follow-up. As a result, 48 patients from the LAGB group were analyzed.

\section{Long-Term Complications}

Complications were classified as "major" or "minor" depending on the need for a surgical re-intervention. All complications that required a re-operation or conversion to another bariatric procedure were classified as "major". All complications that were managed with conservative measures, medication, or were treated under local anesthesia were considered "minor". Major and minor complications are summarized in Table 2.

In both groups, many long-term complications occurred requiring either a re-operation or conversion to another

Table 1 Preoperative patient characteristics

\begin{tabular}{llll}
\hline & LAGB & VBG & $P$ value $^{\mathrm{a}}$ \\
\hline Patients $(n)$ & 50 & 50 & - \\
M/F $(n)$ & $10: 40$ & $10: 40$ & - \\
Age (years) & $39 \pm 8$ & $37 \pm 9$ & 0.32 \\
Weight $(\mathrm{kg})$ & $133 \pm 21$ & $131 \pm 24$ & 0.87 \\
BMI $\left(\mathrm{kg} / \mathrm{m}^{2}\right)$ & $47 \pm 6$ & $47 \pm 6$ & 0.97 \\
Total comorbidities $(n)$ & $1.3 \pm 1.0$ & $1.3 \pm 1.1$ & 0.97 \\
\hline
\end{tabular}

${ }^{\mathrm{a}} t$ test
Table 2 Long-term complications

\begin{tabular}{lcll}
\hline & LAGB & $\begin{array}{l}\text { VBG “non- } \\
\text { converted" } \\
(n=17)\end{array}$ & $\begin{array}{l}\text { RYGB “converted } \\
\text { VBG” } \\
(n=26)\end{array}$ \\
\hline Reflux & $3(6 \%)$ & $1(6 \%)$ & $0(0 \%)$ \\
Vomiting & $20(42 \%)$ & $7(41 \%)$ & $0(0 \%)$ \\
Dysphagia & $1(2 \%)$ & $1(6 \%)$ & $1(4 \%)$ \\
$\begin{array}{l}\text { Port-related } \\
\text { problems }\end{array}$ & $5(10 \%)$ & - & - \\
$\begin{array}{l}\text { Re-operations } \\
\text { Conversions }\end{array}$ & $16(33 \%)$ & $11(26 \%)$ & $2(8 \%)$ \\
Second re- & $5(10 \%)$ & $26(65 \%)$ & - \\
operation & $1(5 \%)$ & $2(8 \%)$ & - \\
\hline
\end{tabular}

bariatric procedure. A flow chart describing the follow-up data including re-operations and conversions is provided in Fig. 1.

\section{Major Complications}

Mean time between the first operation and the re-operation or conversion was 2 years. However, this ranged from a minimum of 6 months to a maximum of 7 years.

Procedure-specific major complications occurred in $60 \%$ of patients after VBG (26/43 patients) and included staple line dehiscence $(n=22)$ and recurrent outlet stenosis $(n=4)$. In all cases, an open conversion to RYGB was performed. Ten patients $(23 \%)$, including four patients who already underwent conversion to RYGB, developed a symptomatic incisional hernia and underwent open repair with mesh. Two patients had complications after the conversion to RYGB necessitating another re-operation (8\%). This included one patient with serious weight regain 2 years after conversion. A distal gastric bypass was performed. Another patient developed a recurrent stenosis of the anastomosis, and a new gastrojejunostomy was created.

Complications necessitating a re-operation after LAGB were also frequent. In total, $44 \%$ (21/48 patients) underwent either re-operation or conversion to another bariatric procedure during follow-up. Main causes were pouch dilatation $(n=10)$, anterior slippage $(n=8)$, band erosion $(n=2)$, and band leakage $(n=1)$. The re-operation rate in perigastric $(n=32)$ and pars flaccida $(n=18)$ operated patients was $41 \%$ and $33 \%$, respectively. Performed laparoscopic re-operations were band refixation (14 patients) and band replacement (two patients). In five patients, refixation or replacement of the band was not possible because of band erosion (two patients) and severe pouch dilatation (three patients). These patients were converted to open biliopancreatic diversion (BPD) in case 
Fig. 1 Flow chart of follow-up, re-operations, and conversions

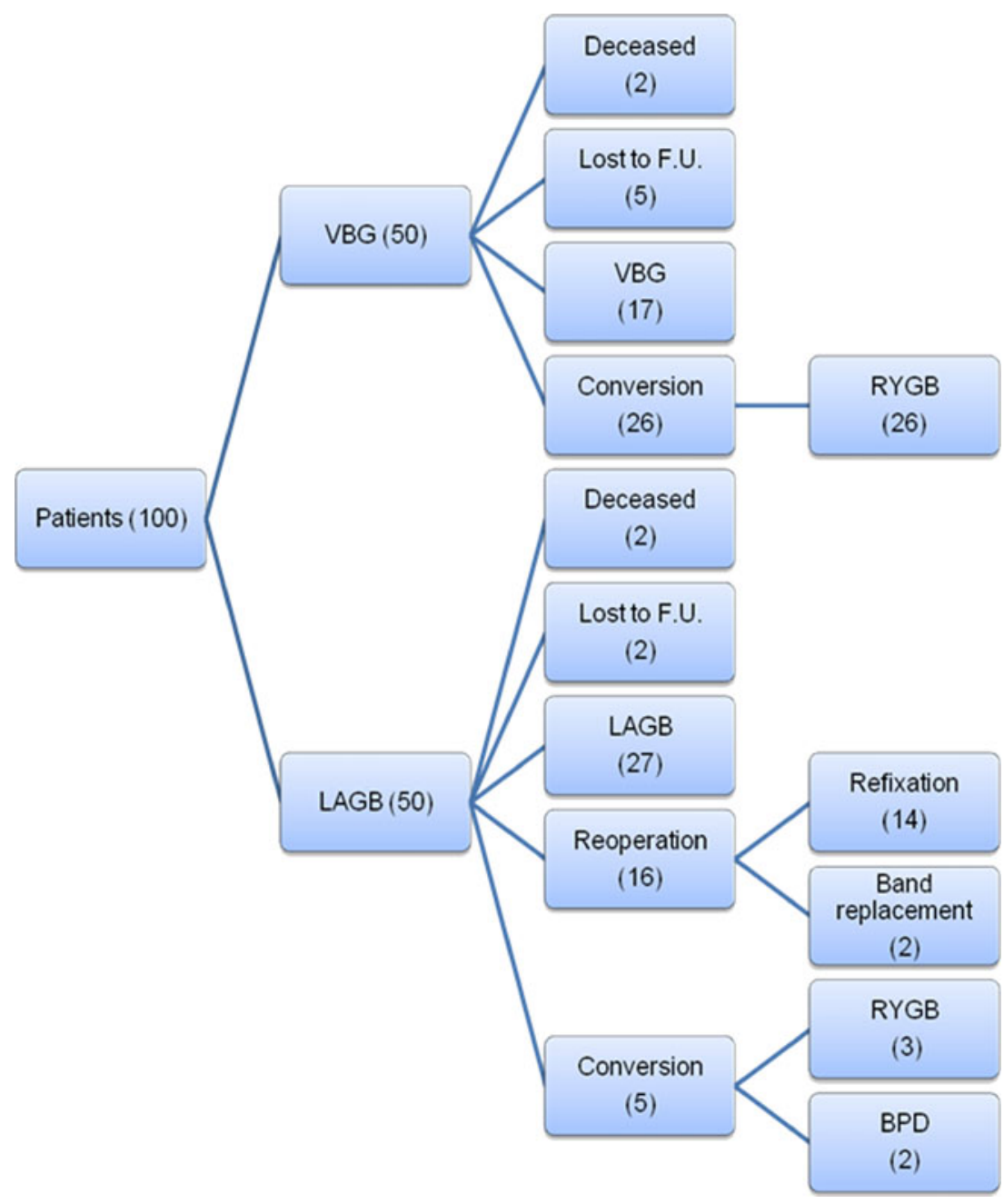

of band erosion or to open RYGB in case of pouch dilatation.

After re-operation, one patient $(1 / 21 ; 5 \%)$ developed a complication which necessitated a second re-operation. This patient had a re-slippage and underwent another laparoscopic refixation.

\section{Minor Complications}

After VBG, minor problems which were still present after 7 years were vomiting (more than once a week; 41\%), reflux (6\%), and dysphagia (6\%). All patients with vomiting more than once a week were patients still having the VBG configuration after 7 years while no patients converted to RYGB complained of vomiting. Reflux and dysphagia occurred in the converted patients in $0 \%$ and $4 \%$, respectively. After LAGB, patients reported the same minor problems: vomiting (42\%), reflux $(6 \%)$, and dysphagia (2\%) without significant differences between uncomplicated and re-operated LAGB patients. Furthermore, 10\% of patients had port-related problems (dislocation in all cases) and were successfully treated under local anesthesia. No port infections occurred.

Weight Loss

\section{$L A G B$ versus $V B G$}

Percent excess weight loss (\% EWL) 2 and 7 years after surgery are represented in Table 3 . In both groups, weight significantly decreased in the first two postoperative years. Weight loss was significantly better after VBG compared with LAGB (EWL of $73 \%$ versus $55 \%$, respectively). In the nonconverted VBG group, a slight but not significant weight gain occurred after 7 years ( $73 \%$ after 2 years and $69 \%$ after 7 years). In the LAGB group, after excluding the five patients that underwent conversion to another bariatric procedure, weight loss remained successful and unchanged after 7 years $(55 \%$ after 2 years and $54 \%$ after 7 years). Furthermore, there was no difference in weight loss between patients with or without reoperation (both EWL of 54\%). However, weight loss remained significantly greater after VBG compared with LAGB. 
Table 3 Weight loss after 2 and 7 years

\begin{tabular}{llll}
\hline & LAGB $(n=48)$ & VBG “non-converted” $(n=17)$ & RYGB “converted VBG” $(n=26)$ \\
\hline BMI (preoperative) & $47 \pm 6(n=50)$ & $43 \pm 7(n=17)$ & $48 \pm 6(n=26)$ \\
BMI (2 years) & $34 \pm 6(n=50)$ & $30 \pm 5(n=36)$ & $33 \pm 7(n=11)$ \\
EWL (2 years) & $55 \% \pm 22(n=49)$ & $73 \% \pm 23(n=36)$ & $66 \% \pm 27(n=11)$ \\
BMI (7 years) & $35 \pm 7(n=43)^{\mathrm{a}}$ & $31 \pm 6(n=17)$ & $31 \pm 5(n=26)$ \\
EWL (7 years) & $54 \% \pm 25(n=43)^{\mathrm{a}}$ & $69 \% \pm 22(n=17)$ & $69 \% \pm 24(n=26)$ \\
\hline
\end{tabular}

${ }^{\mathrm{a}}$ Converted LAGB patients $(n=5)$ were excluded

\section{$V B G$ versus $R Y G B$}

When interpreting long-term weight loss results, it is important to realize that $60 \%$ of VBG patients were converted to RYGB. Preoperative BMI of patients eventually converted to RYGB was significantly higher compared with nonconverted VBG patients (BMI of 48 versus 43 , respectively). Two years after the initial operation, 11 patients were already converted to RYGB. At this point, EWL was not significantly different between converted and non-converted patients $(66 \%$ and $73 \%$, respectively) while after 7 years weight loss was the same in non-converted VBG patients and converted RYGB patients (both EWL of 69\%).

\section{Comorbidities}

In both groups, the most prevalent obesity-related comorbidities were type 2 diabetes mellitus (T2DM), hypertension, and joint problems $(5,7$, and 28 patients in the LAGB group and 7 , 10 , and 29 patients in the VBG group). All obesity-related comorbidities significantly decreased in both groups 2 years after surgery (Table 4). However, an increase in certain comorbidities was observed after 7 years. For example, five patients had T2DM compared with one patient after 2 years. In the VBG group, six patients had hypertension, although only one patient had this after 2 years. Joint problems were again reported by 17 patients in both groups compared with 11 and 12 patients after 2 years in the LAGB and VBG group, respectively. However, the joints involved changed from mainly ankle and knee problems before operation to back problems afterwards. However, in the long-term, the total number of comorbidities per patient decreased after both procedures from 1.3 to $0.6(p=0.01)$. Apart from the increase in T2DM in the LAGB group, there were no significant differences between the two groups. Also, as presented in Table 5, there were no significant differences in the decrease of comorbidities between VBG patients with or without conversion to RYGB $(p=0.8)$.

\section{Laboratory Parameters and Vitamin Status}

Laboratory parameters (hemoglobin, urea, creatinin, ASAT, ALAT, albumin, total protein, cholesterol, triglycerides, HDL, and LDL) and vitamin status (iron, zinc, vitamin B1, B6, B12, and folic acid) 7 years after restrictive surgery are summarized in Table 6. There were no significant differences between patients with LAGB compared with VBG. Furthermore, all values in both groups were within the normal range except high-density lipoprotein which was $2.0 \mathrm{mmol} / \mathrm{L}$ (normal range, $0.7-1.5 \mathrm{mmol} / \mathrm{L}$ ) in the VBG group. Comparing patients with or without conversion to RYGB in the VBG group did not change these results. In Table 7, the laboratory parameters of converted and non-converted VBG patients are listed. No significant differences were found, and no parameters were out of the normal range except HDL (2.2, normal range $0.7-1.5 \mathrm{mmol} / \mathrm{L}$ ) in the converted group.

Noteworthy is that all vitamins were within the normal range in all groups (LAGB, VBG, and RYGB), and no deficiencies were present after 7 years. However, there was a difference in medication use in the form of vitamin supple-

Table 4 Obesity-related comorbidities 2 and 7 years postoperatively (LAGB and VBG patients)

\begin{tabular}{|c|c|c|c|c|c|c|}
\hline & \multicolumn{3}{|l|}{ LAGB } & \multicolumn{3}{|l|}{ VBG } \\
\hline & Preop $(n=50)$ & 2 years $(n=50)$ & 7 years $(n=48)$ & Preop $(n=50)$ & 2 years $(n=48)$ & 7 years $(n=43)$ \\
\hline Diabetes & 5 & 1 & 5 & 7 & 1 & 0 \\
\hline Hypertension & 7 & 3 & 4 & 10 & 1 & 6 \\
\hline Joint problems & 28 & 11 & 17 & 29 & 12 & 17 \\
\hline Hyperchol. & 2 & 1 & 0 & 2 & 1 & 1 \\
\hline OSAS & 1 & 0 & 0 & 1 & 0 & 0 \\
\hline
\end{tabular}


Table 5 Obesity-related comorbidities 2 and 7 years postoperatively (VBG and converted VBG to RYGB patients)

\begin{tabular}{|c|c|c|c|c|c|}
\hline & \multicolumn{3}{|c|}{ VBG “non-converted" $(n=17)$} & \multicolumn{2}{|c|}{ RYGB “converted VBG” $(n=26)$} \\
\hline & Preop $(n=50)$ & 2 years $(n=36)$ & 7 years $(n=17)$ & 2 years $(n=11)$ & 7 years $(n=26)$ \\
\hline Diabetes & 7 & 1 & 0 & 0 & 0 \\
\hline Hypertension & 10 & 1 & 3 & 0 & 3 \\
\hline Joint problems & 29 & 6 & 8 & 6 & 9 \\
\hline Hyperchol. & 2 & 0 & 0 & 1 & 1 \\
\hline OSAS & 1 & 0 & 0 & 0 & 0 \\
\hline
\end{tabular}

ments between the groups. Oral multivitamin supplements were significantly more used by LAGB patients compared with VBG and RYGB patients (55\% versus $32 \%$, respectively). A pattern in vitamin supplement usage and vitamin levels was not apparent. Patients without any supplements also had normal vitamin levels. Vitamin B12 injections (every 3 months) and iron supplements were used more frequently after VBG and RYGB than after LAGB (29\% versus $21 \%$ and $13 \%$ versus $6 \%$ of patients, respectively).

\section{Discussion}

Morbid obesity and its related comorbidities is a worldwide epidemic. Surgery is, in contrast to conventional treatment modalities, the only treatment with a proven long-lasting effect on weight and comorbidities and has a positive effect on the quality of life $[19,20]$. Weight loss more than 5 years after VBG varies in the literature from $30 \%$ to $59 \%$ EWL $[13,14,21]$ while, after LAGB, it varies from $56 \%$ to $62 \%$ EWL $([13,14,22]$. The long-term results of O'Brien et al. show that, 10 years after gastric banding, it is still successful, and weight loss is comparable with gastric bypass (59\% and 52\% EWL, respectively) [23]. Most studies show a rapid weight loss after VBG with a weight regain after 2 years while patients with LAGB tend to lose weight more slowly but for a prolonged period. Our study results are consistent with the literature with $73 \%$ and $55 \%$ EWL after 2 years and $69 \%$ and 54\% EWL after 7 years for VBG and LAGB, respectively. Weight loss was not

Table 6 Laboratory measurements: LAGB versus VBG/GB

\begin{tabular}{|c|c|c|c|c|}
\hline & LAGB $(n=48)$ & VBG $(n=43)$ & Normal range & $P$ value $^{\mathrm{a}}$ \\
\hline Hemoglobin & 8.1 & 7.9 & $7.5-10.0(\mathrm{mmol} / \mathrm{L})$ & 0.61 \\
\hline $\mathrm{ASAT}^{\mathrm{b}}$ & 17 & 20 & <35 (U/L) & 0.13 \\
\hline ALAT $^{\mathrm{c}}$ & 21 & 23 & $<40(\mathrm{U} / \mathrm{L})$ & 0.58 \\
\hline Albumin & 39 & 39 & $35-50(\mathrm{~g} / \mathrm{L})$ & 0.94 \\
\hline Total protein & 69 & 67 & $60-80(\mathrm{~g} / \mathrm{L})$ & 0.49 \\
\hline Cholesterol & 5.2 & 5.0 & $4.3-5.6(\mathrm{mmol} / \mathrm{L})$ & 0.47 \\
\hline Triglycerides & 1.1 & 1.1 & $0.80-2.00(\mathrm{mmol} / \mathrm{L})$ & 0.94 \\
\hline $\mathrm{HDL}^{\mathrm{d}}$ & 1.3 & 2.0 & $0.7-1.5(\mathrm{mmol} / \mathrm{L})$ & 0.22 \\
\hline $\mathrm{LDL}^{\mathrm{e}}$ & 3.5 & 3.0 & $1.9-4.5(\mathrm{mmol} / \mathrm{L})$ & 0.05 \\
\hline Iron & 12.5 & 12.8 & $11.0-30.0(\mu \mathrm{mol} / \mathrm{L})$ & 0.86 \\
\hline Zinc & 12.7 & 12.4 & $10-18(\mu \mathrm{mol} / \mathrm{L})$ & 0.70 \\
\hline Vitamin B1 & 126 & 135 & $74-222(\mathrm{nmol} / \mathrm{L})$ & 0.27 \\
\hline Vitamin B6 & 61 & 78 & 20-122 (nmol/L) & 0.16 \\
\hline Vitamin B12 & 0.23 & 0.27 & $0.15-0.67(\mathrm{nmol} / \mathrm{L})$ & 0.18 \\
\hline Folic acid & 13.9 & 17.5 & $7.0-45.4(\mathrm{mmol} / \mathrm{L})$ & 0.24 \\
\hline
\end{tabular}

${ }^{\mathrm{a}} t$ test

${ }^{\mathrm{b}}$ Aspartate aminotransferase

${ }^{\mathrm{c}}$ Alanine aminotransferase

${ }^{\mathrm{d}}$ High-density lipoprotein

${ }^{\mathrm{e}}$ Low-density lipoprotein 
Table 7 Laboratory measurements after 7 years: VBG versus GB

\begin{tabular}{|c|c|c|c|c|}
\hline & VBG "non-converted" $(n=17)$ & RYGB “converted VBG” $(n=26)$ & Normal range & $P$ value $^{\mathrm{a}}$ \\
\hline Hemoglobin & 7.7 & 7.9 & 7.5-10.0 (mmol/L) & 0.79 \\
\hline $\mathrm{ASAT}^{\mathrm{b}}$ & 18 & 21 & $<35(\mathrm{U} / \mathrm{L})$ & 0.32 \\
\hline ALAT $^{c}$ & 20 & 25 & $<40(\mathrm{U} / \mathrm{L})$ & 0.32 \\
\hline Albumin & 41 & 38 & $35-50(\mathrm{~g} / \mathrm{L})$ & 0.13 \\
\hline Total protein & 68 & 66 & $60-80(\mathrm{~g} / \mathrm{L})$ & 0.58 \\
\hline Cholesterol & 4.8 & 4.9 & $4.3-5.6(\mathrm{mmol} / \mathrm{L})$ & 0.92 \\
\hline Triglycerides & 0.9 & 1.2 & $0.80-2.00(\mathrm{mmol} / \mathrm{L})$ & 0.31 \\
\hline $\mathrm{HDL}^{\mathrm{d}}$ & 1.4 & 2.2 & $0.7-1.5(\mathrm{mmol} / \mathrm{L})$ & 0.32 \\
\hline $\mathrm{LDL}^{\mathrm{e}}$ & 2.9 & 3.0 & $1.9-4.5(\mathrm{mmol} / \mathrm{L})$ & 0.95 \\
\hline Iron & 15.0 & 11.6 & $11.0-30.0(\mu \mathrm{mol} / \mathrm{L})$ & 0.21 \\
\hline Zinc & 12.9 & 12.2 & $10-18(\mu \mathrm{mol} / \mathrm{L})$ & 0.53 \\
\hline Vitamin B1 & 135 & 136 & $74-222(\mathrm{nmol} / \mathrm{L})$ & 0.93 \\
\hline Vitamin B6 & 91 & 69 & 20-122 (nmol/L) & 0.39 \\
\hline Vitamin B12 & 0.27 & 0.26 & $0.15-0.67(\mathrm{nmol} / \mathrm{L})$ & 0.85 \\
\hline Folic acid & 17.5 & 16.1 & $7.0-45.4(\mathrm{mmol} / \mathrm{L})$ & 0.53 \\
\hline
\end{tabular}

${ }^{\mathrm{a}} t$ test

${ }^{\mathrm{b}}$ Aspartate aminotransferase

${ }^{c}$ Alanine aminotransferase

${ }^{\mathrm{d}}$ High-density lipoprotein

${ }^{\mathrm{e}}$ Low-density lipoprotein

significantly different between 2 and 7 years within the groups. Although weight loss was significantly more after VBG compared with LAGB, it is important to notice that $60 \%$ of patients in the VBG group no longer had the VBG configuration but were converted to RYGB because of complications. On the other hand, weight loss was the same after 7 years for patients in the VBG group with or without conversion to RYGB (both EWL of $69 \%$ ). In terms of weight loss, successful bariatric surgery is defined as the maintenance of EWL of at least $50 \%$. In our patient group, this applies to $79 \%$ of VBG patients (converted and nonconverted) and $60 \%$ of LAGB patients.

The decrease in obesity-related comorbidities after bariatric surgery is well-documented and depends on the weight loss and the type of surgery [20, 24-26]. Our study confirms this once again, but interestingly, an increase in T2DM was observed 7 years after LAGB while it was almost nonexistent after 2 years. A clear explanation for this increase from one to five patients was not found. Weight loss in these five patients was EWL $44 \%$ and for the total group of LAGB patients $54 \%$ (ns). They also did not experience a rapid weight gain during the final period of follow-up. Furthermore, three patients in this diabetic group did not have any complications while two patients had a band slippage and underwent refixation. There is no literature available describing a possible increase in diabetes many years after LAGB. Possibly, a combination of factors could be responsible for this phenomenon. The $10 \%$ difference in weight loss could be important because a resolution of T2DM is possible after only $10 \%$ weight loss [27, 28]. Furthermore, the aging of the study population during follow-up could be a factor in the development of T2DM.

A wide range of laboratory measurements was investigated 7 years after surgery including hemoglobin, urea, creatinin, ASAT, ALAT, albumin, total protein, cholesterol, triglycerides, HDL, and LDL. There were no significant differences between the groups and except high-density lipoprotein in the VBG and converted RYGB patients, all values were within normal ranges. The values of minerals and vitamins (iron, zinc, vitamin B1, B6, B12, and folic acid) are especially of interest. In all groups, including the converted RYGB patients, all levels were within the normal range implying no deficiencies were present. In the literature, comparison between restrictive and more malabsorptive procedures shows that deficiencies are much less common after purely restrictive procedures because the gastrointestinal tract remains intact and absorption is normal $[29,30]$. This is confirmed in several reports except that low levels of folic acid are frequently observed caused by a difference in eating habits after surgery (less wholemeal bread, vegetables, and fruit) $[31,32]$. This is considered the most important reason to recommend vitamin supplementation after restrictive surgery. However, in the present study, the postoperative levels of folic acid were normal irrespective of vitamin supplement 
usage. The supplement use was low in both groups but significantly higher in the LAGB group (55\%) compared with the VBG/RYGB group (32\%). Continued vitamin use is probably associated with regular follow-up, and this tends to be higher after LAGB because of visits before and after insufflations. On the other hand, vitamin B12 injections (every 3 months) and iron supplements were used more frequently after VBG and RYGB than after LAGB (29\% versus $21 \%$ and $13 \%$ versus $6 \%$ of patients, respectively). The latter can be explained by the $60 \%$ conversion rate to RYGB in the VBG group. The deficiency of vitamin B12 and iron is well-known after RYGB because of the bypassed stomach and proximal intestine [33-35], and therefore, vitamin monitoring and supplementation is mandatory after RYGB. In our study group, a pattern in vitamin supplement usage and vitamin levels was not apparent. Patients without any supplements also had normal vitamin levels, and this issue has been discussed in the literature [36]. It is mostly explained by medication adherence which is, in case of bariatric surgery, below $50 \%$ and deteriorates during followup $[30,37]$. No randomized trials on this subject are published to provide a definitive answer if supplements are needed after purely restrictive procedures, and therefore, it is still common practice to prescribe vitamin supplements to all bariatric patients. Although our results are not consistent, based on the available literature and possible consequences of deficiencies, continuation of this policy seems justified.

Revisional surgery is often necessary after VBG because of stoma stenosis, pouch enlargement, or stapler line dehiscence. Percentages in the literature range from $10 \%$ to $50 \%$ and are strongly associated with the completeness and duration of follow-up [38-42]. Also, developments in VBG techniques may possibly lead to better results with a lower re-operation rate, e.g., the Mason McLean operation with partition of the stomach instead of only stapling. However, no long-term results of this procedure are described in the literature, so a comparison with standard VBG or LAGB is not possible. In the present study, the mean time between the primary operation and the re-operation was 2 years with a range from 6 months to 7 years. Therefore, in order to present reliable re-operation rates after VBG, a minimum follow-up period of 3 years is necessary. Balsiger et al. described a reoperation rate of $19 \% 10$ years after VBG [21]. Comparable results were found by van de Weijgert et al. with a reoperation rate of $18 \%$ after a mean period of 7.2 years [43]. Studies by van Gemert et al. and MacLean et al. reported higher rates (31\% and $36 \%$, respectively) after 52 months and 2 to 5 years of follow-up [39, 41]. Finally, the prospective study by Miller et al. (follow-up of 92 months) is most comparative with our results with a re-operation rate after VBG of $49.7 \%$ [14]. Purely technical failure after VBG occurred in $65 \%$ of our patients, and in all cases, conversion to RYGB was performed. Conversion from
VBG to RYGB has been described in literature with positive results. Sugerman et al. reported on 53 patients where EWL increased from $36 \%$ to $67 \%$ after the conversion [44]. The patient group of Cordera et al. showed a decrease in BMI of 46 to $35 \mathrm{~kg} / \mathrm{m}^{2}$ after conversion [45]. Kfoury et al. performed a distal RYGB in 45 patients after failed VBG. Results after a follow-up period of at least 2 years were available for 29 patients, and \% EWL was $74 \%$ in this group [46]. Finally, the conversion led to a decrease in BMI from 40 to $32 \mathrm{~kg} / \mathrm{m}^{2}$ at 16 months postoperatively in the group described by Gonzalez et al. [47]. We also reported on 101 patients who were converted from failed VBG to RYGB and demonstrated good results on weight loss, comorbidities, and patients' symptoms [15]. The present study confirms the positive results of this conversion with a long-term EWL of $69 \%$ and a low re-intervention rate $(8 \%)$.

The rate of re-operations after LAGB differs tremendously in literature from $2 \%$ to $80 \%$ [48]. Several reasons for these differences have been proposed. Firstly, results vary according to the used surgical technique, either perigastric or pars flaccida, the latter having better results especially due to a decrease in band slippages [18, 49]. In two randomized studies from Weiner et al. and O'Brien et al., the pars flaccida technique was demonstrated to be a safer technique with less complication and is therefore nowadays considered best surgical practice $[18,49]$. In a previously reported study from our institution based on a study population operated more recently than the population from the present study, the re-operation rate decreased significantly from $42 \%$ to $8 \%$ after introduction of the pars flaccida technique. This was mainly due to a decrease in band slippage and pouch dilatation from $29 \%$ in the perigastric group to $4 \%$ in the pars flaccida group [48]. In the present study, this difference is not confirmed; the re-operation rate after perigastric and pars flaccida operated patients was $41 \%$ and $33 \%$, respectively (ns). However, in this consecutive group of patients, the perigastric technique was used in 30 patients and the pars flaccida technique in 18 patients, the latter representing our first experience with this technique (reviewer no. 1, comment no. 1). Therefore, our experience with the different techniques is not comparable in this group. This might explain the non-significant difference in re-operation rate.

The second important issue explaining differences in reoperation rate after LAGB is follow-up time. In the present study, mean time between the primary operation and the reoperation because of complications after LAGB was 3 years. Most publications report on short-term results after LAGB which explains re-operation rates as low as $2 \%$. However, a follow-up of a reasonable patient population beyond 3 years is necessary for a reliable estimation of the re-operation rate. For example, the re-operation rate in a retrospective study by Christou et al. was $16.1 \%$ after 3 years, but only one third of patients were available for follow-up [50]. In a case- 
controlled study by Cottam et al., the re-operation rate was $53 \%$ in the first year, decreasing to $0 \%$ in the third year. However, follow-up after 3 years was only $25 \%$ [51]. The prospective long-term study by Miller et al., with a follow-up rate of $92 \%$, is therefore the only comparable study with a mean follow-up of 92 months and a minimum of 60 months. They reported a re-operation rate of $8 \%$ which is low compared with the $44 \%$ from the present study [14]. Whether the perigastric or pars flaccida technique was used is unclear. As mentioned above, since the introduction of the pars flaccida technique, our re-operation rate decreased to from $44 \%$ (follow-up 84 months) to $8 \%$ (follow-up of 48 months) [48].

As we previously reported, re-operations after LAGB, especially refixation of the band or replacement, are highly successful [48]. Weiss et al. reported on 73 patients who underwent a surgical revision after LAGB. After a follow-up period of 42 months, BMI decreased from 37.8 to $29.5 \mathrm{~kg} / \mathrm{m}^{2}$ [52]. Peterli et al. performed 250 operations and re-operated on 39 patients $(16 \%)$ because of failed LAGB. After reoperation, $5.3 \%$ of patients developed a second failure of the band, but re-slippages were not observed [53]. These positive results of re-operations after LAGB are confirmed in the present study as only one patient had a re-slippage after refixation of the band (5\%). Weight loss was successful after re-operation and more importantly, the same in patients with or without re-operation (both EWL of 54\%).

In conclusion, VBG results in long-term weight loss and improvement of obesity-related comorbidities. However, because of the $65 \%$ failure rate and the necessity to perform a conversion in case of failure, we do not offer VBG to our patients nowadays. Although the re-operation rate after long-term follow-up is also high in LAGB patients, the results of these re-operations (in most cases only refixation of the band) are good. Many improvements have been made, e.g., the perigastric instead of the pars flaccida technique and low pressure-high volume bands instead of high pressure-low volume bands with a dramatic decrease in re-operation rate as a result. LAGB is the least complicated procedure in comparison with more invasive and non-reversible procedures like RYGB and BPD. Therefore, we still recommend and perform LAGB, but patients need to be informed extensively before the operation and follow-up needs to be durable and complete.

\section{Conclusion}

This long-term follow-up study confirms the high occurrence of late complications after restrictive bariatric surgery. The failure rate of $65 \%$ after VBG is too high, and this procedure is not performed anymore in our institution. The re-operation rate after LAGB is decreasing as a result of new techniques and materials. Results of the re-operations are good with sustained weight loss and reduction in comorbidities. However, in order to achieve these results, durable and complete follow-up after restrictive procedures is imperative.

Conflict of interest The authors declare that they have no conflict of interest.

Open Access This article is distributed under the terms of the Creative Commons Attribution Noncommercial License which permits any noncommercial use, distribution, and reproduction in any medium, provided the original author(s) and source are credited.

\section{References}

1. Mitka M. Surgery for obesity: demand soars amid scientific, ethical questions. Jama. 2003;289(14):1761-2.

2. Tessier DJ, Eagon JC. Surgical management of morbid obesity. Curr Probl Surg. 2008;45(2):68-137.

3. Kemper HC, Stasse-Wolthuis M, Bosman W. The prevention and treatment of overweight and obesity. Summary of the advisory report by the Health Council of The Netherlands. Neth J Med. 2004;62 (1): $10-7$.

4. Bjorntorp P. Results of conservative therapy of obesity: correlation with adipose tissue morphology. Am J Clin Nutr. 1980;33(2 Suppl):370-5.

5. Fisher BL, Schauer P. Medical and surgical options in the treatment of severe obesity. Am J Surg. 2002;184(6B):9S-16.

6. Mason EE et al. Vertical gastroplasty: evolution of vertical banded gastroplasty. World J Surg. 1998;22(9):919-24.

7. Eckhout GV, Willbanks OL, Moore JT. Vertical ring gastroplasty for morbid obesity. Five year experience with 1,463 patients. Am J Surg. 1986;152(6):713-6.

8. Catona A et al. Laparoscopic gastric banding: preliminary series. Obes Surg. 1993;3(2):207-9.

9. van Dielen FM et al. Laparoscopic adjustable gastric banding versus open vertical banded gastroplasty: a prospective randomized trial. Obes Surg. 2005;15(9):1292-8.

10. de Wit LT et al. Open versus laparoscopic adjustable silicone gastric banding: a prospective randomized trial for treatment of morbid obesity. Ann Surg. 1999;230(6):800-5. discussion 805-807.

11. Olbers $\mathrm{T}$ et al. Randomized clinical trial of laparoscopic Roux-en$\mathrm{Y}$ gastric bypass versus laparoscopic vertical banded gastroplasty for obesity. Br J Surg. 2005;92(5):557-62.

12. Morino $\mathrm{M}$ et al. Laparoscopic adjustable silicone gastric banding versus vertical banded gastroplasty in morbidly obese patients: a prospective randomized controlled clinical trial. Ann Surg. 2003;238(6):835-41. discussion 841-842.

13. Nilsell $\mathrm{K}$ et al. Prospective randomised comparison of adjustable gastric banding and vertical banded gastroplasty for morbid obesity. Eur J Surg. 2001;167(7):504-9.

14. Miller K, Pump A, Hell E. Vertical banded gastroplasty versus adjustable gastric banding: prospective long-term follow-up study. Surg Obes Relat Dis. 2007;3(1):84-90.

15. Schouten $\mathrm{R}$ et al. Conversion of vertical banded gastroplasty to Roux-en-Y gastric bypass results in restoration of the positive effect on weight loss and co-morbidities: evaluation of 101 patients. Obes Surg. 2007;17(5):622-30.

16. Belachew $\mathrm{M}$ et al. Laparoscopic adjustable gastric banding. World J Surg. 1998;22(9):955-63. 
17. Dargent J. Pouch dilatation and slippage after adjustable gastric banding: is it still an issue? Obes Surg. 2003;13(1):111-5.

18. Weiner $\mathrm{R}$ et al. A prospective randomized trial of different laparoscopic gastric banding techniques for morbid obesity. Surg Endosc. 2001;15(1):63-8.

19. Karlsson $J$ et al. Ten-year trends in health-related quality of life after surgical and conventional treatment for severe obesity: the SOS intervention study. Int J Obes (Lond). 2007;31(8):1248-61.

20. Sjostrom L et al. Lifestyle, diabetes, and cardiovascular risk factors 10 years after bariatric surgery. N Engl J Med. 2004;351 (26):2683-93.

21. Balsiger BM et al. Ten and more years after vertical banded gastroplasty as primary operation for morbid obesity. J Gastrointest Surg. 2000;4(6):598-605.

22. O'Brien PE, Dixon JB. Lap-band: outcomes and results. J Laparoendosc Adv Surg Tech A. 2003;13(4):265-70.

23. O'Brien PE et al. Systematic review of medium-term weight loss after bariatric operations. Obes Surg. 2006;16(8):1032-40.

24. Buchwald $\mathrm{H}$ et al. Bariatric surgery: a systematic review and meta-analysis. Jama. 2004;292(14):1724-37.

25. Chapman AE et al. Laparoscopic adjustable gastric banding in the treatment of obesity: a systematic literature review. Surgery. 2004;135(3):326-51.

26. Colquitt J. et al. Surgery for morbid obesity. Cochrane Database Syst Rev. 2005(4):CD003641.

27. Pi-Sunyer $X$ et al. Reduction in weight and cardiovascular disease risk factors in individuals with type 2 diabetes: one-year results of the look AHEAD trial. Diab Care. 2007;30(6):1374-83.

28. Knowler WC et al. Reduction in the incidence of type 2 diabetes with lifestyle intervention or metformin. N Engl J Med. 2002;346 (6):393-403.

29. Pournaras DJ, le Roux CW. After bariatric surgery, what vitamins should be measured and what supplements should be given? Clin Endocrinol (Oxf). 2009;71(3):322-5.

30. Coupaye $\mathrm{M}$ et al. Nutritional consequences of adjustable gastric banding and gastric bypass: a 1-year prospective study. Obes Surg. 2009;19(1):56-65.

31. Gasteyger $\mathrm{C}$ et al. Changes in body composition, metabolic profile and nutritional status 24 months after gastric banding. Obes Surg. 2006;16(3):243-50.

32. Cooper PL et al. Nutritional consequences of modified vertical gastroplasty in obese subjects. Int J Obes Relat Metab Disord. 1999;23(4):382-8.

33. Parkes E. Nutritional management of patients after bariatric surgery. Am J Med Sci. 2006;331(4):207-13.

34. Malinowski SS. Nutritional and metabolic complications of bariatric surgery. Am J Med Sci. 2006;331(4):219-25.

35. Fujioka K. Follow-up of nutritional and metabolic problems after bariatric surgery. Diab Care. 2005;28(2):481-4.

36. Ledoux $\mathrm{S}$ et al. Comparison of nutritional consequences of conventional therapy of obesity, adjustable gastric banding, and gastric bypass. Obes Surg. 2006;16(8):1041-9.
37. Trostler $\mathrm{N}$ et al. Nutrient intake following vertical banded gastroplasty or gastric bypass. Obes Surg. 1995;5(4):403-10.

38. Hall JC et al. Gastric surgery for morbid obesity. The Adelaide Study. Ann Surg. 1990;211(4):419-27.

39. van Gemert WG et al. Revisional surgery after failed vertical banded gastroplasty: restoration of vertical banded gastroplasty or conversion to gastric bypass. Obes Surg. 1998;8(1): 21-8.

40. Nightengale ML et al. Prospective evaluation of vertical banded gastroplasty as the primary operation for morbid obesity. Mayo Clin Proc. 1991;66(8):773-82.

41. MacLean LD, Rhode BM, Forse RA. Late results of vertical banded gastroplasty for morbid and super obesity. Surgery. 1990;107(1):20-7.

42. Naslund E et al. Seven year results of vertical banded gastroplasty for morbid obesity. Eur J Surg. 1997;163(4):281-6.

43. van de Weijgert EJ, Ruseler CH, Elte JW. Long-term follow-up after gastric surgery for morbid obesity: preoperative weight loss improves the long-term control of morbid obesity after vertical banded gastroplasty. Obes Surg. 1999;9(5):426-32.

44. Sugerman HJ et al. Conversion of failed or complicated vertical banded gastroplasty to gastric bypass in morbid obesity. Am J Surg. 1996;171(2):263-9.

45. Cordera $\mathrm{F}$ et al. Unsatisfactory weight loss after vertical banded gastroplasty: is conversion to Roux-en-Y gastric bypass successful? Surgery. 2004;136(4):731-7.

46. Kfoury E, Vanguri A. Distal Roux-en-Y gastric bypass conversion operation for failed vertical banded gastroplasty. Obes Surg. 1993;3(1):41-3.

47. Gonzalez R et al. Operative technique for converting a failed vertical banded gastroplasty to Roux-en-Y gastric bypass. J Am Coll Surg. 2005;201(3):366-74.

48. Schouten R, van Dielen FM, Greve JW. Re-operation after laparoscopic adjustable gastric banding leads to a further decrease in BMI and obesity-related co-morbidities: results in 33 patients. Obes Surg. 2006;16(7):821-8.

49. O'Brien PE et al. A prospective randomized trial of placement of the laparoscopic adjustable gastric band: comparison of the perigastric and pars flaccida pathways. Obes Surg. 2005;15 (6):820-6.

50. Christou N, Efthimiou E. Five-year outcomes of laparoscopic adjustable gastric banding and laparoscopic Roux-en-Y gastric bypass in a comprehensive bariatric surgery program in Canada. Can J Surg. 2009;52(6):E249-58.

51. Cottam DR et al. A case-controlled matched-pair cohort study of laparoscopic Roux-en-Y gastric bypass and Lap-Band patients in a single US center with three-year follow-up. Obes Surg. 2006;16 (5):534-40.

52. Weiss $\mathrm{HG}$ et al. Surgical revision after failure of laparoscopic adjustable gastric banding. Br J Surg. 2004;91(2):235-41.

53. Peterli $\mathrm{R}$ et al. Re-operations following laparoscopic adjustable gastric banding. Obes Surg. 2002;12(6):851-6. 